\title{
Twelve-thousand laser-AO observations: first results from the Robo-AO large surveys
}

\author{
Nicholas M. Law ${ }^{a}$; Christoph Baranec ${ }^{b}$; Reed L. Riddle ${ }^{c}$ \\ ${ }^{a}$ Department of Physics and Astronomy, University of North Carolina at Chapel Hill, Chapel \\ Hill, NC 27599-3255, USA \\ ${ }^{b}$ Institute for Astronomy, University of Hawai'i at Mānoa, Hilo, HI 96720-2700, USA \\ ${ }^{c}$ Division of Physics, Mathematics, and Astronomy, California Institute of Technology, \\ Pasadena, CA, 91125, USA
}

\begin{abstract}
Robo-AO is the first AO system which can feasibly perform surveys of thousands of targets. The system has been operating in a fully robotic mode on the Palomar $1.5 \mathrm{~m}$ telescope for almost two years. Robo-AO has completed nearly 12,000 high-angular-resolution observations in almost 20 separate science programs including exoplanet characterization, field star binarity, young star binarity and solar system observations. We summarize the Robo-AO surveys and the observations completed to date. We also describe the data-reduction pipeline we developed for Robo-AO - the first fully-automated AO data-reduction, point-spread-function subtraction and companion-search pipeline.
\end{abstract}

Keywords: astronomy, adaptive optics, robotic, Robo-AO, exoplanet characterization, surveys

\section{INTRODUCTION}

Robo-AO is an autonomous laser-guide-star adaptive-optics (AO) and science instrument ${ }^{3}$ currently deployed on the 60-inch telescope at Palomar Observatory (Figure 1). Robo-AO's robotic high-efficiency observing capability allows it to observe thousands of astronomical targets per month at the visible diffraction limit (Figure 2).

Further author information: Send correspondence to N.M.L.; nmlaw@physics.unc.edu

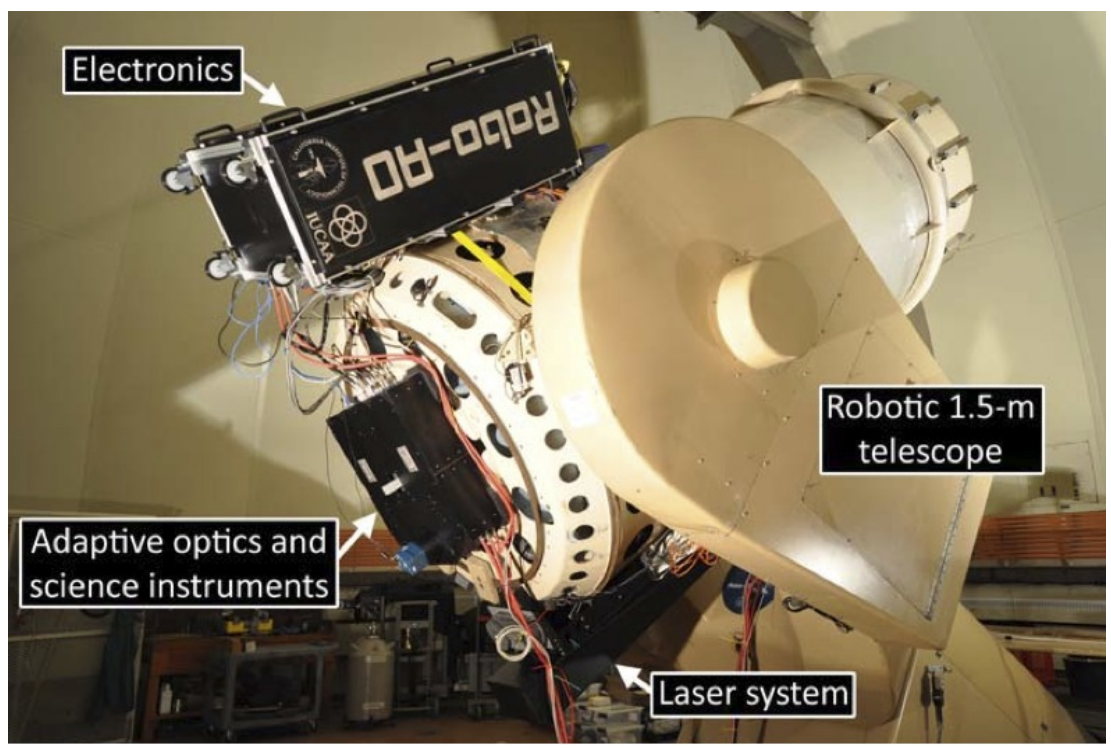

Figure 1. The Robo-AO instrument, consisting of a Cassegrain-mounted adaptive optics system and visible-light science camera, a side-mounted electronics rack, and a side-mounted 355nm laser launch telescope. 


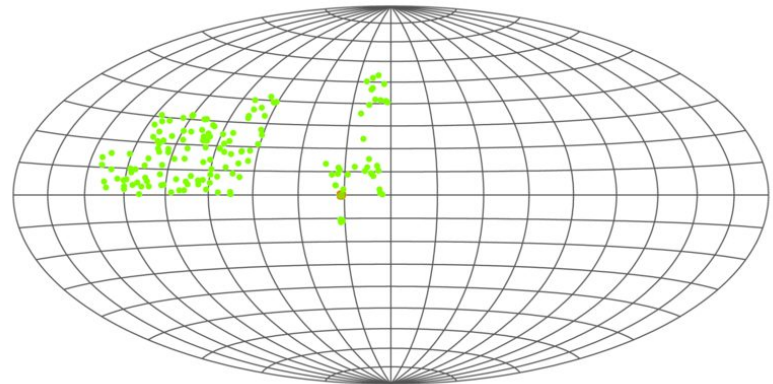

2012/10/03

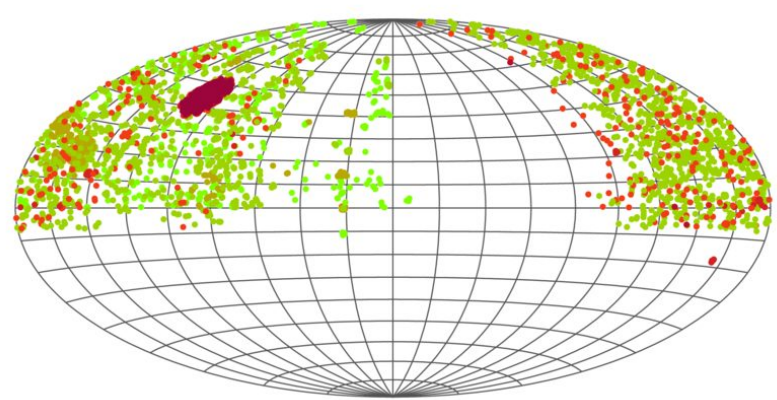

$2013 / 08 / 14$

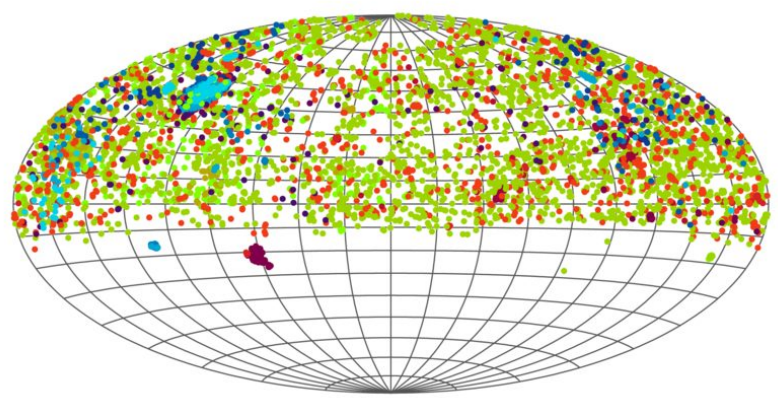

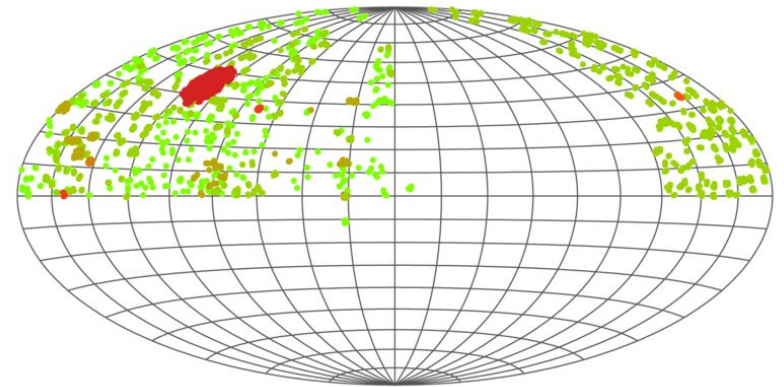

$2013 / 01 / 21$

6764 observations

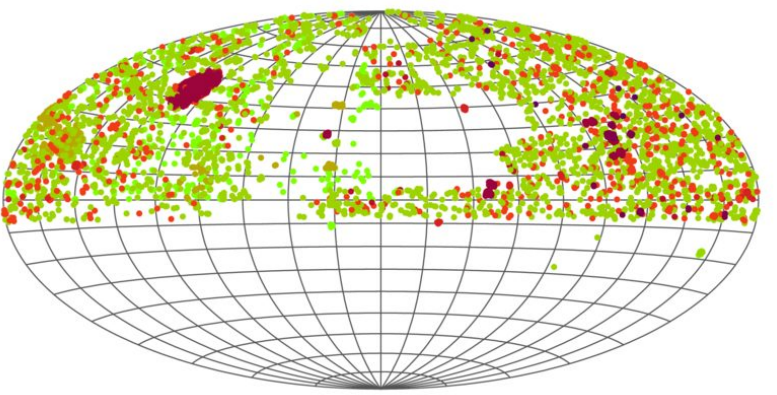

$2014 / 03 / 25$

11572 observations
19 projects

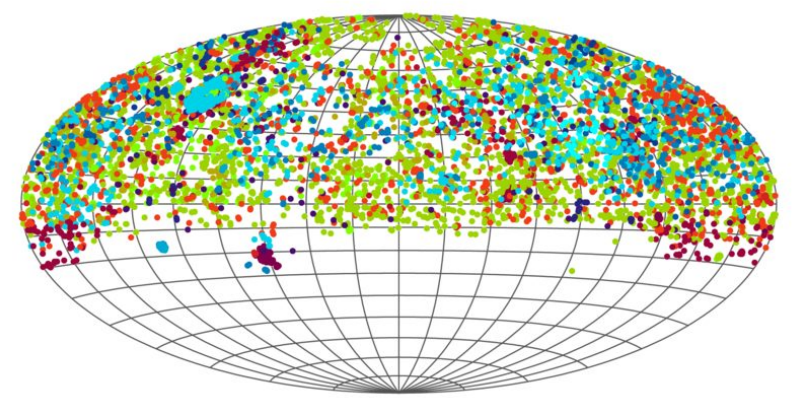

Figure 2. High-angular-resolution visible-light AO imaging performed by Robo-AO, as a function of time. Each image shows the cumulative number of targets Robo-AO had observed across the sky, at selected observing nights from the first fully-robotic operations in Summer 2012 through to the latest observing run (as of the time of writing) in March 2014. Different observing programs are denoted by different colors and many targets and observing programs overlap; the large red / cyan area in the top left of each plot is the Kepler field. Each of the 11,572 points on these graphs is an observation which was performed by the robot and automatically processed by the pipeline into a final science-quality image which passed our quality checks. 
The prototype system routinely performs visible-light imaging ${ }^{1,2}$ with angular resolutions approaching the diffraction limit of a $1.5-\mathrm{m}$ telescope, $\approx 0.12 "$. Robotic software automations ${ }^{4}$ keep target-to-target observing overheads to less than 1.5 minutes (including slew time) typically leading to twenty 90 -s observations per hour (over two-hundred targets per night). Since the start of fully-robotic operations in Summer 2012 Robo-AO has made almost 12,000 observations for 19 separate science programs.

In this paper we describe the Robo-AO science programs (Section 2) and the automated AO data reduction system used to reduce data for them (Section 3). We summarize in Section 4.

\section{ROBO-AO SURVEY PROGRAMS AND SURVEY EFFICIENCY}

Robo-AO has observed on 79 nights since Summer 2012 (with additional nights lost to weather). Robo-AO observes with a queue-scheduling system (see Riddle et al. 2014, these proceedings). Multiple programs are executed each night depending on target availability. The Robo-AO observing program is broken up into key projects, which received much of the observing time in the first year of operation, and individual smaller projects proposed by members of the astronomical community via the Caltech and JPL Time Allocation Committees.

\subsection{Robo-AO Major Projects}

The Robo-AO Kepler-Field Survey. Using Robo-AO we have already conducted the largest-ever imaging survey of Kepler exoplanet hosts, detecting 53 companions around 715 stars in the first season of our observations ${ }^{5}$ (Figure 3). We expect to complete observations of every Kepler planet candidate in 2014.

This survey, when complete, will yield a comprehensive assessment of the stellar companion population around every planet candidate in the Kepler survey; constraints on false positives among the Kepler planet candidate population; and a detailed measurement of the effects of stellar multiplicity on planetary system architecture. Our first-season results are already showing tentative evidence for these effects (Figure 4).

Also among the discoveries in this initial dataset are several instances in which apparently secure detections of multiple planets around a single star (probabilistically validated by nature of their multiplicity ${ }^{6,7}$ ) turn out, upon adaptive optics imaging, to likely be some combination of planets around multiple stars within the same Kepler pixels.

We also observe selected Kepler-field targets outside of our comprehensive program, searching for close companions to Kepler planet candidates ${ }^{8,9}$ and exotic systems, ${ }^{10}$ as well as providing separated photometry for close binaries in the Kepler field. ${ }^{11}$

The Robo-AO Nearby-Star Survey. Our nearby-star binarity survey is aimed at producing the most comprehensive and homogenous measurement of stellar multiplicity in the solar neighbourhood. Its two main thrusts are a volume-limited survey of every star within $25 \mathrm{pc}$ (using the RECONS database ${ }^{12}$ ) and a magnitudelimited survey of M-dwarf multiplicity within 33pc. Both surveys are targeted at the nearby, bright stars that will be the targets of the next generation of radial-velocity, direct imaging, and transit exoplanet surveys. The observations for both surveys are complete; the volume-limited survey has observed 1,220 nearby stars of all stellar types, while the magnitude-limited survey has observed 1,918 nearby-M-dwarfs covering a wide range of masses and metallicities.

\subsection{Other Robo-AO projects}

Robo-AO has also performed many smaller projects, with less than a few hundred targets each. The queue scheduled operation makes it simple to submit prioritized target lists with hundreds or thousands of observations, which are then performed at the most efficient times over the following months. The largest programs in this category are:

Exoplanet \& planetary formation follow-up: Robo-AO is searching for stellar companions around exoplanet hosts ${ }^{13}$ (PI: J. Desert); verifying debris-disk stars selected from infrared surveys (PI: S. Hinkley); and following-up transiting exoplanet candidates from the PTF M-dwarfs survey ${ }^{14}$ (PI: N. Law).

Field stellar binarity: Robo-AO is searching for high-order multiple systems in solar-type binaries (PIs: R. Riddle / A. Tokovinin); high-order multiplicity in extremely wide binary systems (PI: S. Dhital); binarity in 

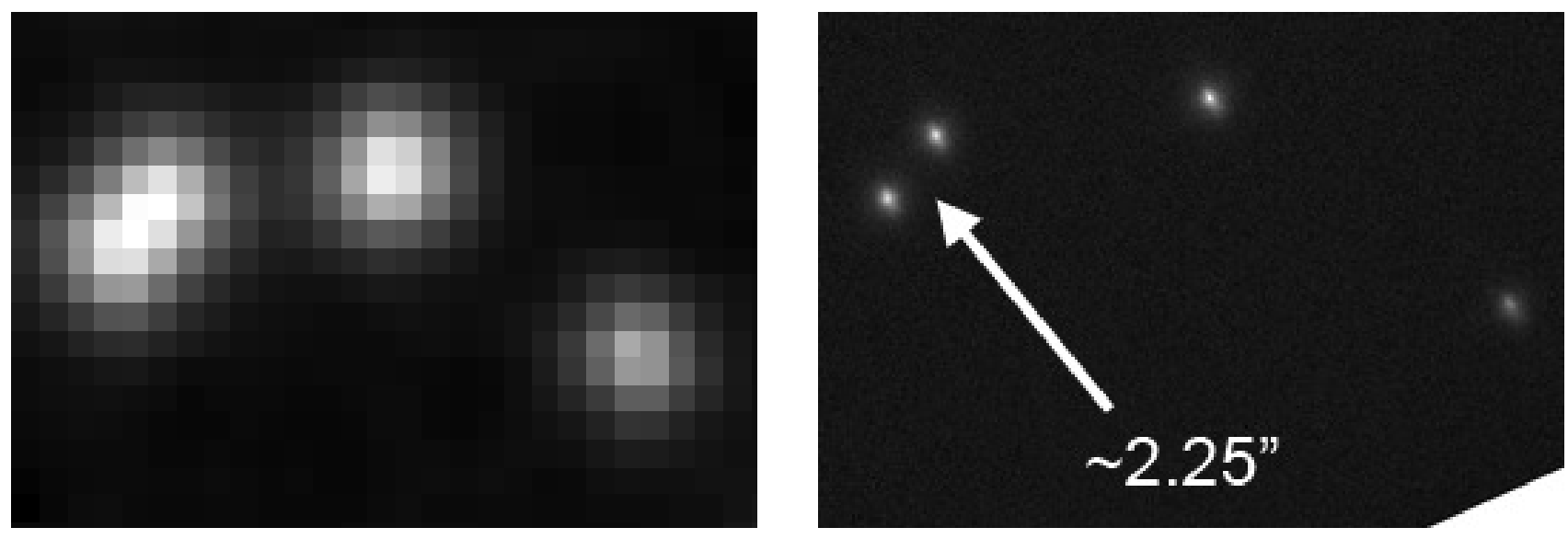

Figure 3. an example field around a Kepler planet candidate, comparing between observed at the seeing limit (left, taken from the Digital Sky Survey) and Robo-AO observations (right).

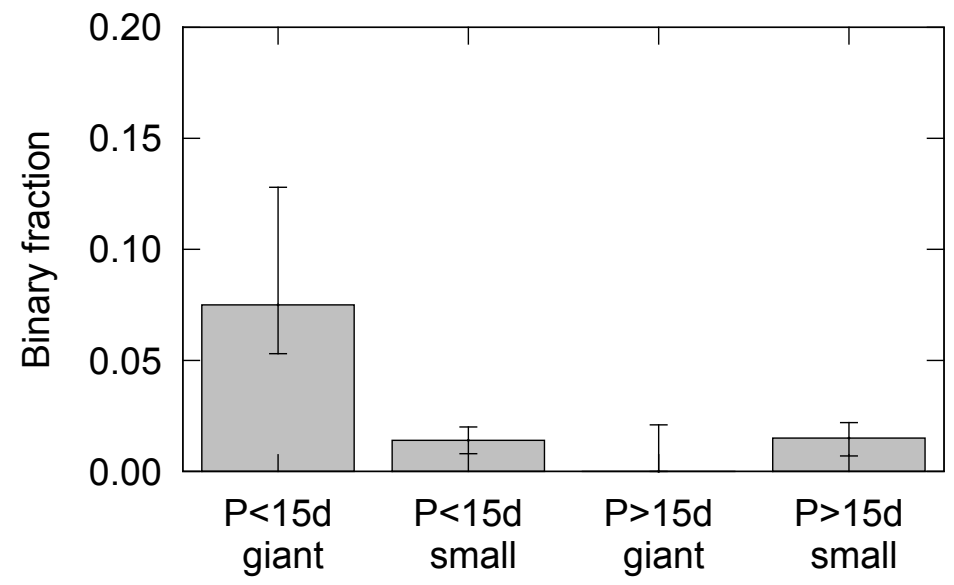

Figure 4. Fraction of Kepler planet candidates with Robo-AO detected nearby stars for four different planetary populations. Giant here is shorthand for a radius equal to or larger than that of Neptune. We assign Kepler planet candidates to these populations if any planet in the system meets the requirements; a small number of multiple-planet systems are therefore assigned to multiple populations. We only include the most likely physically-associated systems in this chart; see Law et al. $2014^{5}$ for details.

UV-bright M-dwarfs (PIs: E. Gaidos / R. Riddle); and the system has been used to confirm binaries found using weak-lensing techniques in wide-field imaging ${ }^{15}$ (PI: N. Law).

Young stellar binarity: There are several ongoing surveys for pre-main-sequence and young-star binarity in clusters ${ }^{16}$ of various ages (PIs: J. Curtis, R. Riddle, L. Hillenbrand).

Solar system science: Robo-AO's improved angular resolution has allowed the monitoring of photometric variability in comet ISON with dramatically-reduced interference from the cometary tail (PI: M. Drahus), as well improved-resolution imaging of other solar system objects (Figure 5).

Extragalactic \& high-energy science: Robo-AO has provided high-angular resolution imaging for several supernova events; is assessing the binarity of AGN nuclei (PI: S. Tendulkar); and is evaluating high-precision astrometric techniques to search for intermediate-mass black holes in globular cluster cores (PI: S. Hildebrandt). 


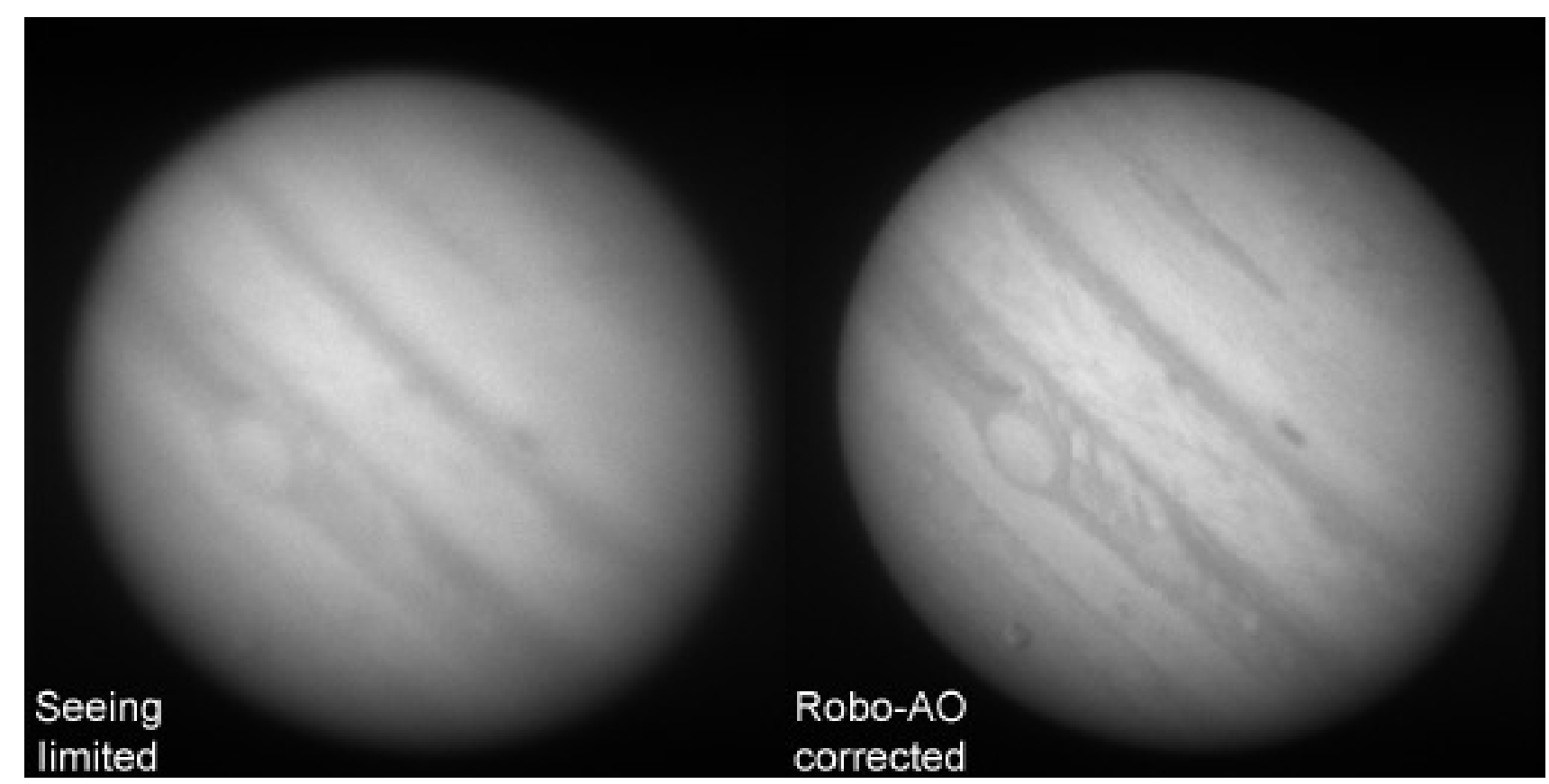

Figure 5. Robo-AO observations of Jupiter in the r' filter. The cloud detail and contrast are much improved compared to the seeing-limited image taken within a few minutes of the AO image. Note the detail visible in the AO image of of the moon Ganymede, silhouetted against Jupiter's clouds and barely visible in the seeing-limited image.

\section{AUTOMATED AO DATA REDUCTION}

Individual Robo-AO observations are currently made with an electron multiplying CCD camera with a 44" square field of view and 0.043 " pixel scale. The camera is read out continually at a frame rate of $8.6 \mathrm{~Hz}$ during science observations, allowing image motion (which cannot be measured using the laser system) to be removed in software after observations with the presence of a $m_{V} \leq 16$ guide star within the field of view. During typical observing, where objects are generally scattered around the sky, we generally obtain residual wavefront errors in the 160 to $200 \mathrm{~nm}$ RMS range, leading to the ability to detect and characterize stellar companions at contrasts of $>5$ magnitudes at separations of $0.25-1$ arcseconds at visible wavelengths (and down to separations of $\approx 0.1$ arcseconds).

The raw data for a typical Robo-AO observation consists of several thousand $1024 \times 1024$ frames recorded in a FITS cube. In almost all cases the individual frames must be aligned and stacked for science use. We designed and built an automated data reduction pipeline which automatically reduces all the science data taken with Robo-AO. Reduced and calibrated science images are typically available within minutes of the data being taken by Robo-AO.

Figure 6 shows the detailed data flow though the pipeline. The pipeline proceeds in several major steps; many science programs only utilize the first frame alignment and stacking step, but the Kepler and nearby-star major programs also utilize the PSF subtraction, companion detection and characterization steps.

Frame stacking and alignment: The pipeline automatically selects the brightest star in the observed data using a down-sampled image generated from a stack of the entire observation. The system selects a small (few-arcsecond) region to use as the guide region. For each frame, that region is extracted and then bi-cubically upsampled by a factor of four to allow sub-pixel registration. The upsampled image is then cross-correlated with a simulated diffraction-limited point spread function to search for the brightest peak; the cross-correlation improves the signal-to-noise of the location measurement compared to the raw image. ${ }^{17}$ The individual frames are dark-subtracted and flat fielded (using calibration images automatically taken each afternoon). The frames are then aligned and stacked using the Drizzle algorithm ${ }^{18}$ with a standard factor-of-two up-sampling. 
1. Robo-AO

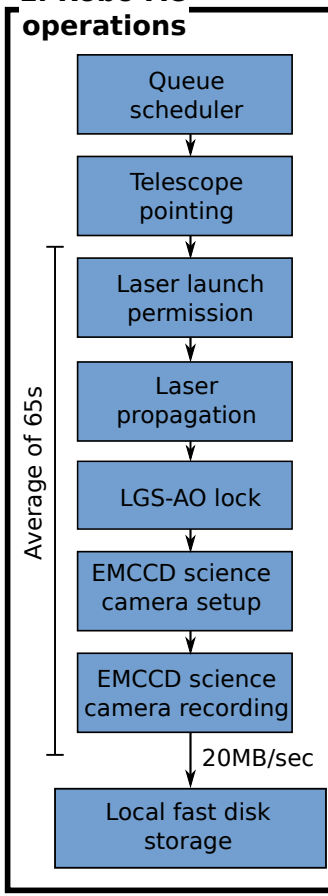

Typical performance

\section{Observing}

0-60 seconds pointing

65 seconds LGS-AO operations

Negligable camera deadtime

Image pipeline

Reduction at $\sim$ speed of data collection

Images complete reduction within 10 mins. of data collection

PSF subtraction

2-5 minutes per image

Companion detection

1-2 minutes per image

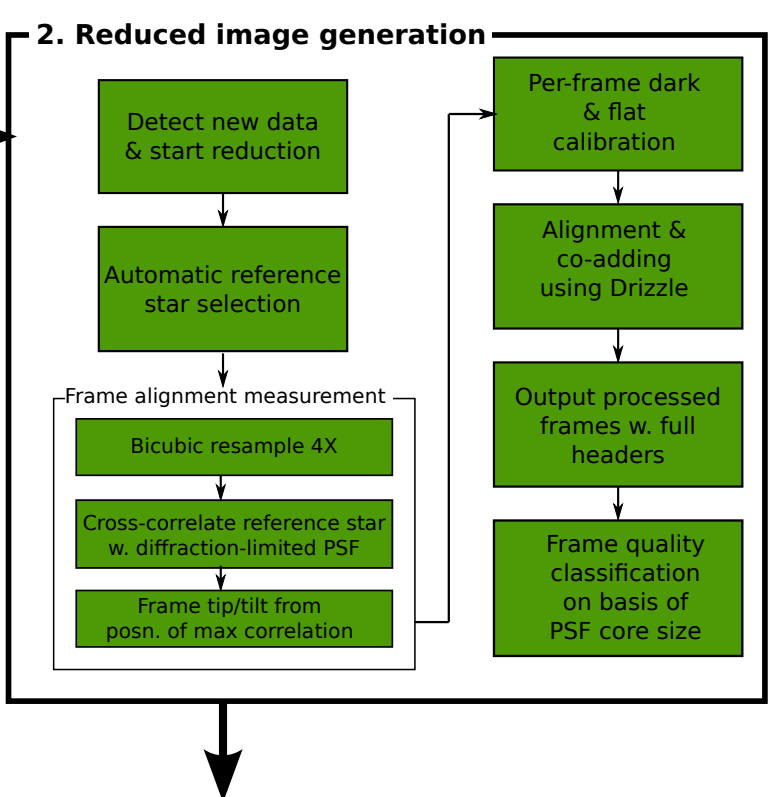

3. Locally-optimized PSF subtraction

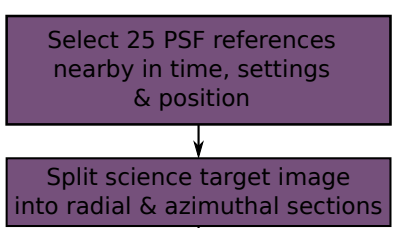

$\downarrow$

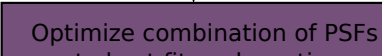

to best fit each section

$\downarrow$

Subtract best-fit PSF
4. Companion detection $\&$ characterization

Cross-correlate PSF-subtracted image w. diffraction-limited PSF

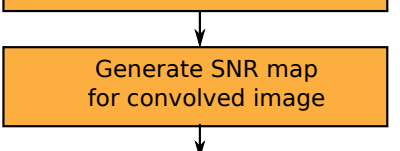

,

Flag significant peaks in SNR map as possible companions

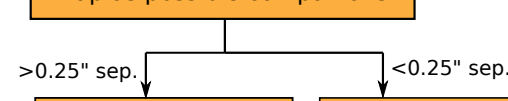

Aperture photometry Gaussian-fit astrometry astrometry and photometry

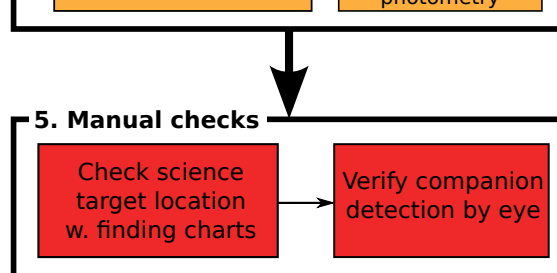

Figure 6. The Robo-AO data reduction pipeline. The Robo-AO operations section has been greatly simplified; see Baranec et al. 2014 and Riddle et al. 2014, these proceedings, for the details. The PSF subtraction and companion detection subsystems are run only for science programs which require them. 
PSF subtraction and companion characterization: The PSF subtraction and companion detection algorithms are described in detail elsewhere. ${ }^{5}$ The PSF subtraction takes advantage of the large number of observations Robo-AO takes each night; this provides a wide variety of observations to be used as PSF references. The subtraction itself is a custom locally-optimized PSF subtraction routine based on the LOCI algorithm (Locally Optimized Combination of Images ${ }^{19}$ ), and typically achieves photon-noise-limited performance for the standard few-minute Robo-AO observations.

The companion detection algorithms are based on a novel approach to automatic robust companion detection, described in detail in Law et al. 2014. ${ }^{5}$ We first measure the local image noise as a function of distance from the target star. We then use the noise fits to estimate the significance of each pixel's signal level above the locally-estimated noise. This procedure generates a significance image where bright pixels in regions of high photon noise (i.e. in the core of the star) are down-weighted compared to those in lower-noise areas. We then cross-correlate the significance image with a Gaussian corresponding to the diffraction limit of the Robo-AO observation. We then select the pixels which show the most significant detections $(>5 \sigma)$, and amalgamate groups of multiple significant pixels into single companion detections. The companion properties (contrast ratio, separation, position angle) are then automatically measured.

Manual checks: After these automated procedures complete the only manual steps in the process occur: 1) checks that the system locked onto the correct science target for guiding, PSF subtraction and companion detection (crowded fields like the Kepler field often have several bright stars); and 2) a manual check that the detected companions are not spurious. Typically only a few percent of the final pipeline results need further investigation or changes.

\section{SUMMARY}

Robo-AO has completed almost two years of fully-autonomous laser-guide-star adaptive optics operation. The system routinely and reliably observes over 200 targets each night at 0.12 -arcsecond resolutions in visible light, and has completed almost 12,000 high-angular-resolution observations. Six refereed science papers have been published, and the results from more than a dozen further science programs are in preparation.

\section{ACKNOWLEDGMENTS}

The Robo-AO system is supported by collaborating partner institutions, the California Institute of Technology and the Inter-University Centre for Astronomy and Astrophysics, by the National Science Foundation under Grant Nos. AST-0906060 and AST-0960343, by the Mount Cuba Astronomical Foundation, and by a gift from Samuel Oschin. We are grateful to the Palomar Observatory staff for their ongoing support of Robo-AO on the 60-inch telescope, particularly S. Kunsman, M. Doyle, J. Henning, R. Walters, G. Van Idsinga, B. Baker, K. Dunscombe and D. Roderick. C.B. acknowledges support from the Alfred P. Sloan Foundation.

\section{REFERENCES}

[1] Baranec, C., Riddle, R., Ramaprakash, A. N., Law, N., Tendulkar, S., Kulkarni, S., Dekany, R., Bui, K., Davis, J., Burse, M., Das, H., Hildebrandt, S., Punnadi, S., and Smith, R., "Robo-AO: autonomous and replicable laser-adaptive-optics and science system," in [Society of Photo-Optical Instrumentation Engineers (SPIE) Conference Series], Society of Photo-Optical Instrumentation Engineers (SPIE) Conference Series 8447 (July 2012).

[2] Baranec, C., Riddle, R., Law, N. M., Ramaprakash, A. N., Tendulkar, S. P., Bui, K., Burse, M. P., Chordia, P., Das, H. K., Davis, J. T. C., Dekany, R. G., Kasliwal, M. M., Kulkarni, S. R., Morton, T. D., Ofek, E. O., and Punnadi, S., "Bringing the Visible Universe into Focus with Robo-AO," Journal of Visual Experiments 72, 50021 (Feb. 2013).

[3] Baranec, C., Riddle, R., Law, N. M., Ramaprakash, A. N., Tendulkar, S. P., Hogstrom, K., Bui, K., Burse, M. P., Chordia, P., Das, H. K., Dekany, R. G., Kulkarni, S. R., and Punnadi, S., "High-efficiency autonomous laser adaptive optics," ApJL in press, (2014). 
[4] Riddle, R. L., Burse, M. P., Law, N. M., Tendulkar, S. P., Baranec, C., Rudy, A. R., Sitt, M., Arya, A., Papadopoulos, A., Ramaprakash, A. N., and Dekany, R. G., "The Robo-AO software: fully autonomous operation of a laser guide star adaptive optics and science system," in [Society of Photo-Optical Instrumentation Engineers (SPIE) Conference Series], Society of Photo-Optical Instrumentation Engineers (SPIE) Conference Series 8447 (July 2012).

[5] Law, N. M., Morton, T., Baranec, C., Riddle, R., Ravichandran, G., Ziegler, C., Johnson, J. A., Tendulkar, S. P., Bui, K., Burse, M. P., Das, H. K., Dekany, R. G., Kulkarni, S., Punnadi, S., and Ramaprakash, A. N., "Robotic Laser-Adaptive-Optics Imaging of 715 Kepler Exoplanet Candidates using Robo-AO," ArXiv:1312.4958 (Dec. 2013).

[6] Lissauer, J. J., Marcy, G. W., Rowe, J. F., Bryson, S. T., Adams, E., Buchhave, L. A., Ciardi, D. R., Cochran, W. D., Fabrycky, D. C., Ford, E. B., Fressin, F., Geary, J., Gilliland, R. L., Holman, M. J., Howell, S. B., Jenkins, J. M., Kinemuchi, K., Koch, D. G., Morehead, R. C., Ragozzine, D., Seader, S. E., Tanenbaum, P. G., Torres, G., and Twicken, J. D., "Almost All of Kepler's Multiple-planet Candidates Are Planets," ApJ 750, 112 (May 2012).

[7] Rowe, J. F., Bryson, S. T., Marcy, G. W., Lissauer, J. J., Jontof-Hutter, D., Mullally, F., Gilliland, R. L., Issacson, H., Ford, E., Howell, S. B., Borucki, W. J., Haas, M., Huber, D., Steffen, J. H., Thompson, S. E., Quintana, E., Barclay, T., Still, M., Fortney, J., Gautier, III, T. N., Hunter, R., Caldwell, D. A., Ciardi, D. R., Devore, E., Cochran, W., Jenkins, J., Agol, E., Carter, J. A., and Geary, J., "Validation of Kepler's Multiple Planet Candidates. III. Light Curve Analysis and Announcement of Hundreds of New Multi-planet Systems," ApJ 784, 45 (Mar. 2014).

[8] Swift, J. J., Johnson, J. A., Morton, T. D., Crepp, J. R., Montet, B. T., Fabrycky, D. C., and Muirhead, P. S., "Characterizing the Cool KOIs. IV. Kepler-32 as a Prototype for the Formation of Compact Planetary Systems throughout the Galaxy," ApJ 764, 105 (Feb. 2013).

[9] Muirhead, P. S., Becker, J., Feiden, G. A., Rojas-Ayala, B., Vanderburg, A., Price, E. M., Thorp, R., Law, N. M., Riddle, R., Baranec, C., Hamren, K., Schlawin, E., Covey, K. R., Johnson, J. A., and Lloyd, J. P., "Characterizing the Cool KOIs. VI. H- and K-band Spectra of Kepler M Dwarf Planet-Candidate Hosts," ApJS 21352014 (June 2014).

[10] Muirhead, P. S., Vanderburg, A., Shporer, A., Becker, J., Swift, J. J., Lloyd, J. P., Fuller, J., Zhao, M., Hinkley, S., Pineda, J. S., Bottom, M., Howard, A. W., von Braun, K., Boyajian, T. S., Law, N., Baranec, C., Riddle, R., Ramaprakash, A. N., Tendulkar, S. P., Bui, K., Burse, M., Chordia, P., Das, H., Dekany, R., Punnadi, S., and Johnson, J. A., "Characterizing the Cool KOIs. V. KOI-256: A Mutually Eclipsing Post-common Envelope Binary," ApJ 767, 111 (Apr. 2013).

[11] Montet, B., Johnson, J. A., Muirhead, P. S., Shporer, A., Howard, A., Baranec, C., Albert, L., and The Robo-AO Collaboration, "LHS 6343: Precise Constraints on the Mass and Radius of a Transiting Brown Dwarf Discovered by Kepler," in [American Astronomical Society Meeting Abstracts \#223], American Astronomical Society Meeting Abstracts 223, \#334.03 (Jan. 2014).

[12] Henry, T. J., Jao, W.-C., Subasavage, J. P., Beaulieu, T. D., Ianna, P. A., Costa, E., and Méndez, R. A., "The Solar Neighborhood. XVII. Parallax Results from the CTIOPI 0.9 m Program: 20 New Members of the RECONS 10 Parsec Sample," AJ 132, 2360-2371 (Dec. 2006).

[13] Thorp, R., Desert, J., Baranec, C., Law, N. M., Johnson, J. A., and Riddle, R. L., "Study of the Impact of Stellar Multiplicity on Planet Occurrence and Properties," in [American Astronomical Society Meeting Abstracts \#223], American Astronomical Society Meeting Abstracts 223, \#152.16 (Jan. 2014).

[14] Law, N. M., Kraus, A. L., Street, R., Fulton, B. J., Hillenbrand, L. A., Shporer, A., Lister, T., Baranec, C., Bloom, J. S., Bui, K., Burse, M. P., Cenko, S. B., Das, H. K., Davis, J. T. C., Dekany, R. G., Filippenko, A. V., Kasliwal, M. M., Kulkarni, S. R., Nugent, P., Ofek, E. O., Poznanski, D., Quimby, R. M., Ramaprakash, A. N., Riddle, R., Silverman, J. M., Sivanandam, S., and Tendulkar, S. P., "Three New Eclipsing White-dwarf-M-dwarf Binaries Discovered in a Search for Transiting Planets around M-dwarfs," ApJ 757, 133 (Oct. 2012).

[15] Terziev, E., Law, N. M., Arcavi, I., Baranec, C., Bloom, J. S., Bui, K., Burse, M. P., Chorida, P., Das, H. K., Dekany, R. G., Kraus, A. L., Kulkarni, S. R., Nugent, P., Ofek, E. O., Punnadi, S., Ramaprakash, A. N., Riddle, R., Sullivan, M., and Tendulkar, S. P., "Millions of Multiples: Detecting and Characterizing Close-separation Binary Systems in Synoptic Sky Surveys," ApJS 206, 18 (June 2013). 
[16] Curtis, J. L. and Wright, J., "Rotation and activity at 3 Gyr with Ruprecht 147," in [American Astronomical Society Meeting Abstracts \#223], American Astronomical Society Meeting Abstracts 223, \#442.08 (Jan. 2014).

[17] Law, N. M., Mackay, C. D., and Baldwin, J. E., "Lucky imaging: high angular resolution imaging in the visible from the ground," A\&A 446, 739-745 (Feb. 2006).

[18] Fruchter, A. S. and Hook, R. N., "Drizzle: A Method for the Linear Reconstruction of Undersampled Images," PASP 114, 144-152 (Feb. 2002).

[19] Lafrenière, D., Marois, C., Doyon, R., Nadeau, D., and Artigau, É., "A New Algorithm for Point-Spread Function Subtraction in High-Contrast Imaging: A Demonstration with Angular Differential Imaging," ApJ 660, 770-780 (May 2007). 